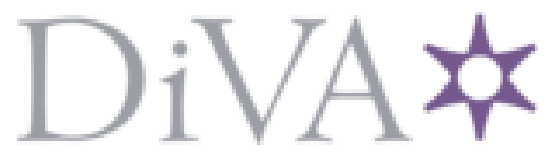

http://www.diva-portal.org

This is the published version of a paper published in Proceedings of the Combustion Institute.

Citation for the original published paper (version of record):

Magri, L., See, Y-C., Tammisola, O., Ihme, M., Juniper, M. (2016)

Multiple-scale thermoacoustic stability analysis of a coaxial jet combustor.

Proceedings of the Combustion Institute

https://doi.org/10.1016/j.proci.2016.06.009

Access to the published version may require subscription.

N.B. When citing this work, cite the original published paper.

Permanent link to this version:

http://urn.kb.se/resolve?urn=urn:nbn:se:kth:diva-198180 
Available online at www.sciencedirect.com

ScienceDirect

Proceedings of the Combustion Institute 000 (2016) 1-9

Proceedings of the

Combustion

Institute

\title{
Multiple-scale thermo-acoustic stability analysis of a coaxial jet combustor
}

\author{
L. Magri ${ }^{\text {ad, }}{ }^{\text {,* }}$, Y.-C. See ${ }^{\text {b }}$, O. Tammisola ${ }^{c}$, M. Ihme ${ }^{\text {a }}$, M.P. Juniper ${ }^{d}$

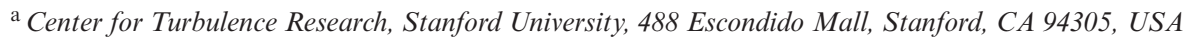 \\ b Convergent Science, Madison, WI 53719, USA \\ ${ }^{\mathrm{c}}$ Mechanical, Materials and Manufacturing Engineering, University of Nottingham, Nottingham NG7 2 RD, UK \\ ${ }^{\mathrm{d}}$ Cambridge University Engineering Department, Trumpington Street, Cambridge CB2 1PZ, UK
}

Received 3 December 2015; accepted 1 June 2016

Available online xxx

\begin{abstract}
In this paper, asymptotic multiple-scale methods are used to formulate a mathematically consistent set of thermo-acoustic equations in the low-Mach number limit for linear stability analysis. The resulting sets of nonlinear equations for hydrodynamics and acoustics are two-way coupled. The coupling strength depends on which multiple scales are used. The double-time-double-space (2T-2S), double-time-single-space (2T-1S) and single-time-double-space (1T-2S) limits are revisited, derived and linearized. It is shown that only the 1T$2 \mathrm{~S}$ limit produces a two-way coupled linearized system. Therefore this limit is adopted and implemented in a finite-element solver. The methodology is applied to a coaxial jet combustor. By using an adjoint method and introducing the intrinsic sensitivity, (i) the interaction between the acoustic and hydrodynamic subsystems is calculated and (ii) the role of the global acceleration term, which is the coupling term from the acoustics to the hydrodynamics, is analyzed. For the confined coaxial jet diffusion flame studied here, (i) the growth rate of the thermo-acoustic oscillations is found to be more sensitive to small changes in the hydrodynamic field around the flame and (ii) increasing the global acceleration term is found to be stabilizing in agreement with the Rayleigh Criterion.
\end{abstract}

(C) 2016 by The Combustion Institute. Published by Elsevier Inc.

Keywords: Thermo-acoustics; Stability; Adjoint methods; Multiple-scale analysis; Sensitivity analysis

\section{Introduction}

Thermo-acoustic oscillations in gas turbines and rocket engines can lead to catastrophic failure

\footnotetext{
* Corresponding author at: Center for Turbulence Research, Stanford University, 488 Escondido Mall, Stanford, CA 94305, USA. Fax: +1 6507253525.

E-mail address: 1magri@stanford.edu (L. Magri).
}

and are one of the most persistent problems facing engine manufacturers [1]. They arise when acoustic pressure fluctuations occur sufficiently in phase with heat-release fluctuations. This converts thermal energy to mechanical energy over a cycle [2].

Thermo-acoustic oscillations are governed by the interaction between two macro subsystems: the combusting hydrodynamics and the acoustics. The hydrodynamics determines the 
flow field around the flame. The acoustics excites hydrodynamic structures at the flame base, which are then convected downstream, causing heat-release fluctuations. Although it has been argued that hydrodynamic instability has little influence on the thermo-acoustic stability, experiments [3] showed that the frequency of the coupled hydrodynamic/thermo-acoustic system is dictated by the hydrodynamic mechanism at some operating conditions and the thermo-acoustic mechanism at others. The interactions between the two subsystems is still not fully understood.

Linear stability analysis is often used to predict whether a combustion system will experience thermo-acoustic oscillations. The hydrodynamic subsystem is typically modeled by a response function [e.g. 4,5], or a time-delayed model [e.g. 1], relating the heat release to the acoustic velocity. These models are coupled with low-order acoustic solvers, such as network models or Helmholtz solvers [6], and the eigenvalues are calculated. If at least one eigenvalue has positive growth rate, the system is unstable. The sensitivity of the stability to external feedback mechanisms or passive control has been calculated with adjoint-based methods [7-9], which were recently experimentally validated [10]. However, these versatile methods were applied only to simplified thermo-acoustic models with uniform mean flows.

In most thermo-acoustic models, the interaction from the acoustics to the hydrodynamics is globally simulated by a simplified convection model, such as the $n-\tau$ model, meaning that the hydrodynamic variables are not included in the state vector. The system is only one-way coupled. To overcome this limitation, multiple-scale methods were applied by [11-13] to capture the two-way interaction between the hydrodynamics and acoustics. [11] used one time and two spatial scales (1T-2S) to couple a one-dimensional acoustic network model with a one-dimensional low-Mach number flame solver. Using the same multiple scales, [12] studied the nonlinearities in an electrical Rijke-tube with onedimensional acoustics. The effect that the acoustics has on the hydrodynamics was assumed to be uniform in the hydrodynamic domain. [13] used two time and two spatial scales (2T-2S) in nonlinear simulations of a backward-facing step diffusion flame combustor to investigate the role of the acoustic Reynolds stress.

The main contribution of this paper is to develop and apply a three-dimensional thermoacoustic linear model able to capture the two-way coupling between the hydrodynamics and acoustics. We apply the method to an axisymmetric coaxial jet combustor [14-16] with a diffusion flame. The chemistry is modeled by a one-step reaction but could be extended to detailed chemistry models. The intrinsic sensitivity is calculated with an adjoint method. This quantifies the growth rate's dependence on every interaction between the two subsys- tems. This directly gives the drift in the dominant eigenvalue caused by the global acceleration term, which couples the acoustics to the hydrodynamics, avoiding time-consuming nonlinear simulations [12]. The ultimate aim is to set up a robust framework for stability, receptivity and sensitivity analysis of combustion-acoustic systems and thereby to control thermo-acoustic instabilities by exploiting the behavior of hydrodynamic instabilities.

\section{Governing equations}

The non-dimensional compressible continuity, momentum, and energy equations are

$$
\begin{aligned}
& \frac{\partial \rho}{\partial \tau}+\left(\frac{L}{h} M\right) \nabla \cdot(\rho \mathbf{u})=0, \\
& \rho \frac{\partial \mathbf{u}}{\partial \tau}+\left(\frac{L}{h} M\right)\left[\rho \mathbf{u} \cdot \nabla \mathbf{u}+\frac{1}{\gamma M^{2}} \nabla p-\frac{1}{S_{1} R e} \nabla \cdot \overline{\boldsymbol{\tau}}\right] \\
& \quad=0,
\end{aligned}
$$

$$
\begin{aligned}
& \rho \frac{\partial T}{\partial \tau}+\left(\frac{L}{h} M\right)\left[\rho \mathbf{u} \cdot \nabla T-\frac{1}{S_{1} \operatorname{RePr}} \Delta T\right. \\
& \left.-\rho D a Q_{R}+\frac{\gamma-1}{\gamma} \mathbf{u} \cdot \nabla p\right]+\frac{\gamma-1}{\gamma} \frac{\partial p}{\partial \tau}=0,
\end{aligned}
$$

where $\rho$ is the density scaled by the ambient (oxidizer) density, $\tilde{\rho}_{0}$; $\mathbf{u}$ is the velocity scaled by the inlet fuel velocity, $\tilde{u}_{f} ; p$ is the pressure scaled by the ambient pressure, $\tilde{p}_{0} ; T$ is the temperature nondimensionalized as $\left(\tilde{T}-\tilde{T}_{0}\right) /\left(\tilde{T}_{f}-\tilde{T}_{0}\right)$, where $\tilde{T}_{f}$ is the adiabatic flame temperature and $\tilde{T}_{0}$ is the ambient oxidizer temperature; $Q_{R}$ is the rate of heat released by reaction; $M$ is the Mach number; $\gamma$ is the heat capacity ratio; $R e$ is the Reynolds number based on fuel parameters, $\tilde{\rho}_{f} \tilde{u}_{f} h / \tilde{\mu}$, with $\tilde{\mu}$ being the dynamic viscosity; $S_{1}$ is the oxidizer-tofuel density ratio, $\tilde{\rho}_{0} / \tilde{\rho}_{f} ; \operatorname{Pr}$ is the Prandtl number $\tilde{\mu} \tilde{c}_{p} / \tilde{\lambda}$, where $\tilde{c}_{p}$ is the heat capacity at constant pressure and $\tilde{\lambda}$ is the thermal conductivity; $D a$ is the Damköhler number $(\gamma-1) \tilde{\omega}_{0} h /\left(\gamma \tilde{u}_{0}\right) ; \tilde{\omega}_{0}$ is the reference reaction rate. We assume the Newtonian constitutive relation for the viscous stress tensor, $\overline{\boldsymbol{\tau}}=\nabla \mathbf{u}+(\nabla \mathbf{u})^{T}-2 / 3(\nabla \cdot \mathbf{u}) \mathbf{I}$, where $\mathbf{I}$ is the identity tensor. The reference length is the mean flame length, $h$, upstream of which it is assumed that most of the heat is released by chemical reaction. The flame acts on the acoustics as a compact heat source of finite spatial extent. The reference time is $L / \tilde{c}_{0}$, where $\tilde{c}_{0}$ is the reference speed of sound and $L$ is the length of the combustor (Fig. 1). $\tau$ is the dimensionless acoustic time. We have neglected body forces and viscous dissipation effects in the energy equation, as a result of the low-Mach number limit. We have assumed Fourier's law for conduction, constant thermo-viscous properties, and a 


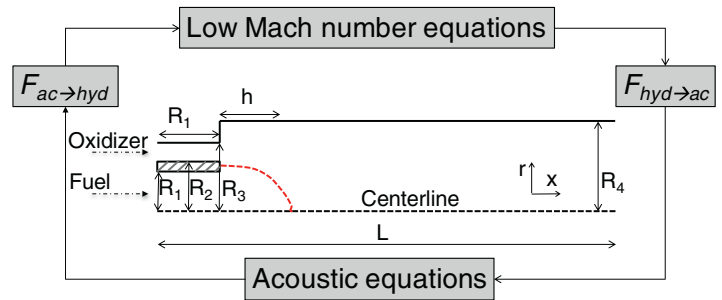

Fig. 1. Coupling between combusting hydrodynamics, governed by the low-Mach number equations, and acoustics. Depending on the multiple-scale limit, the coupling terms, $F_{a c \rightarrow h y d}$ and $F_{h y d} \rightarrow a c$, have different expressions (Table 1). The schematic shows the coaxial jet combustor. $R_{1}=3.157 \mathrm{~cm}, R_{2}=3.175 \mathrm{~cm}, R_{3}=4.685 \mathrm{~cm}, R_{4}=6.115 \mathrm{~cm}, L=9 R_{1}$. The curved dashed line represents the stoichiometric surface. In the computations, all the spatial variables are scaled by the fuel jet diameter, $2 R_{1}$.

perfect gas law. We study diffusion flames with onestep chemistry by defining the mixture fraction, $Z$, and using the non-dimensional Arrhenius' law

$$
\begin{aligned}
\rho \frac{\partial Z}{\partial \tau} & +\left(\frac{L}{h} M\right)\left[\rho \mathbf{u} \cdot \nabla Z-\frac{1}{S_{1} \operatorname{ReSc}} \Delta Z\right]=0 \\
Q_{R}= & \rho^{2} \omega \\
= & \rho^{2}\left[\left(Z-\frac{T}{s+1}\right)\left(1-Z-\frac{s T}{s+1}\right)-\kappa T^{2}\right] \\
& \times \exp \left[\frac{-\beta(1-T)}{1-\alpha(1-T)}\right],
\end{aligned}
$$

where $S c$ is the Schmidt number; $\tilde{\mu} /\left(\tilde{\rho}_{f} \tilde{D}\right)$, with $\tilde{D}$ being the mass diffusivity; $s$ is the stoichiometric ratio; $\kappa$ is the equilibrium constant; $\alpha=1-\tilde{T}_{f} / \tilde{T}_{0} ; \beta$ is the Zeldovich number, $\alpha \tilde{T}_{a} / \tilde{T}_{0}$, where $\tilde{T}_{a}$ is the activation energy; the Lewis number is assumed to be unity and $\tilde{\rho} \tilde{D}$ is constant. The state equation becomes $\rho\left[\left(S_{1}-1\right) Z+1\right]\left[\left(S_{2}-1\right) T+1\right]-p=0$, where $S_{2}$ is the adiabatic-flame-to-ambient temperature ratio. These equations, with slightly different non-dimensionalization, were also used in $[17,18]$.

\section{Two-way coupling of hydrodynamics and acoustics}

We couple the reacting low-Mach number equations, which govern hydrodynamic phenomena, with the acoustic equations in a mathematically consistent manner by combining an asymptotic approach with a multiple-scale method. This enables us to reduce the complexity of the full problem governed by (1)-(5). In thermo-acoustics, multiplescale methods were applied, among others, by [1113]. The two perturbation parameters are the Mach number, $M \sim O(\epsilon)$ and the flame compactness, $h / L \sim O\left(\epsilon^{n}\right)$, where $0<\epsilon \ll 1$. The Mach number is the smallest perturbation parameter, hence 0 $\leq n \leq 1$. In most gas turbine chambers, acoustic phenomena evolve at scales that are different from those of hydrodynamic phenomena. This is because low-frequency thermo-acoustic instabilities are expected to scale with the longitudinal length, $L$, whereas hydrodynamic instabilities are expected to scale with the flame length or shear-layer thickness, $h$. High-frequency transverse instabilities are not considered in this multiple-scale analysis. We define $t$ as the hydrodynamic time, $\mathbf{x}$ as the hydrodynamic spatial coordinates, and $\boldsymbol{\xi}$ as the acoustic spatial coordinates. Observing that hydrodynamic phenomena scale with the convective time, $h / \tilde{u}_{0}$, and flame length, $h$; and acoustic phenomena scale with the acoustic time, $L / \tilde{c}_{0}$, and combustor's length, $L$, it follows that $t / \tau=M L / h=\epsilon^{1-n}$ and $x_{i} / \xi_{i}=L / h=\epsilon^{-n}$. Physically, this means that we require $\epsilon^{(n-1)} \geq 1$ acoustic time units, $\tau$, to obtain one convective time unit, $t$. In such a case the acoustic time is faster than the convective time. Likewise, we require $\epsilon^{n} \leq 1$ acoustic spatial units, $\boldsymbol{\xi}$, to obtain one convective spatial unit, $\mathbf{x}$. In such a case the acoustic space is longer than the convective space. We show and discuss three multiple-scale limits.

(i) In the double-time-double-space limit (2T$2 \mathrm{~S})$, the acoustics evolve at shorter time scales and longer spatial scales than the hydrodynamics. The description of this approach is given by [13], who carried out nonlinear simulations of diffusion flames in a backward-facing step dump combustor. In this case $0<n<1$, i.e., the perturbation coefficient is strictly positive and smaller than unity.

(ii) In the double-time-single-space limit (2T1S), the acoustics evolve at shorter time scales but at the same spatial scale as the hydrodynamics. The derivation is provided by [19]. In this case $n=0$, i.e., there are no different spatial scales.

(iii) In the single-time-double-space limit (1T$2 \mathrm{~S})$, the acoustics evolves at longer spatial scales but at the same time scale as the hydrodynamics. In this case $n=1$, i.e., there are no different time scales. The derivation for nonreacting atmospheric flows is given by [20]; for a one-dimensional acoustic system with a flame by [11]; and for an electrical Rijke tube by [12]. 


\subsection{Multiple-scale method}

To reduce the complexity and separate out hydrodynamic and acoustic phenomena from the original Eqs. (1)-(5), we carry out the following procedure.

(i) Asymptotic expansion: We expand the variables assuming a low-Mach number decomposition of the form $\phi=\Sigma_{i} \epsilon^{i} \phi_{i}$, where $\phi$ denotes a generic variable.

(ii) Differential operators decomposition: In the double-time-double-space approach (2T-2S), $\phi(\mathbf{x}, t) \rightarrow \phi(\mathbf{x}, \xi, t, \tau)$. By applying the chain rule we decompose both the temporal and spatial derivatives as $\partial / \partial \tau$ $\rightarrow \partial / \partial \tau+\epsilon^{1-n} \partial / \partial t$, and $\nabla \rightarrow \nabla_{x}+\epsilon^{n} \nabla_{\xi}$. In the double-time-single-space approach (2T-1S), $\phi(\mathbf{x}, t) \rightarrow \phi(\mathbf{x}, t, \tau)$ and only the temporal derivative is decomposed as $\partial / \partial \tau$ $\rightarrow \partial / \partial \tau+\epsilon^{1-n} \partial / \partial t$. In the single-timedouble-space approach (1T-2S), $\phi(\mathbf{x}, t) \rightarrow$ $\phi(\mathbf{x}, \xi, t)$ and only the spatial derivative is decomposed as $\nabla \rightarrow \nabla_{x}+\epsilon^{n} \nabla_{\xi}$.

(iii) Order-by-order matching: New equations are defined by collecting terms in order of $\epsilon$.

(iv) Average-plus-fluctuation decomposition and equation averaging: In $2 \mathrm{~T}-2 \mathrm{~S}$, the time decomposition $\phi=\langle\phi\rangle_{\tau}+\phi^{\prime \tau}$, is substituted into the operator presented in (ii) and the equations are time averaged over the slow hydrodynamic time scale $t$. The angle brackets $\langle\cdot\rangle_{\tau}$ represent the time average of the fast variable, $\tau$, the superscript ' $\tau$ represents the fluctuation over the fast time $\tau$. Then, the variables are split as $\phi=\langle\phi\rangle_{x}+\phi^{\prime x}$, and the equations are spatially averaged over the long acoustic spatial scale $\xi$. The angle brackets $\langle\cdot\rangle_{x}$ represent the spatial average of the short spatial variable $\mathbf{x}$, the superscript ' $x$ represents the fluctuation over the short spatial scale $\mathbf{x}$. In $2 \mathrm{~T}-1 \mathrm{~S}$, only the time decomposition and averaging is applied. In 1T-2S, only the spatial decomposition and averaging is applied.

Regardless of the limit used, the above four steps lead to a nonlinearly coupled set of lowMach number and acoustic equations, which are explained in Sections 3.2 and 3.3.

\subsection{Hydrodynamic phenomena: the low-Mach number equations}

Hydrodynamic phenomena are governed by the continuity, momentum, energy and mixturefraction low-Mach number equations for constant pressure flames [21]

$\frac{\partial \rho}{\partial t}+\nabla_{x} \cdot(\rho \mathbf{u})=0$,

$$
\begin{aligned}
& \frac{\partial \mathbf{u}}{\partial t}+\mathbf{u} \cdot \nabla_{x} \mathbf{u}+\frac{1}{\gamma \rho} \nabla_{x} p-\frac{1}{S_{1} \operatorname{Re} \rho} \nabla_{x} \cdot \overline{\boldsymbol{\tau}}=F_{a c \rightarrow h y d}, \\
& \frac{\partial T}{\partial t}+\mathbf{u} \cdot \nabla_{x} T-\frac{1}{S_{1} \operatorname{RePr} \rho} \Delta_{x} T-D a Q_{R}=0, \\
& \frac{\partial Z}{\partial t}+\mathbf{u} \cdot \nabla_{x} Z-\frac{1}{S_{1} \operatorname{ReSc\rho }} \Delta_{x} Z=0,
\end{aligned}
$$

where the spatial gradient $\nabla_{x}$ acts on the hydrodynamic spatial scale, $\mathbf{x}$. The state equation is $\rho\left[\left(S_{1}-1\right) Z+1\right]\left[\left(S_{2}-1\right) T+1\right]=1$, which shows that the thermodynamic pressure is constant and equal to unity when non-dimensionalized. This nonlinear problem can be conveniently expressed in matrix form as $\dot{\mathbf{q}}_{\text {hyd }}-\mathbf{H}\left(\mathbf{q}_{h y d}\right)=\mathbf{F}(\mathbf{q})_{a c \rightarrow h y d}$, where $\mathbf{q}_{h y d}=(\rho, \mathbf{u}, T, Z)^{T}$ is the vector of the hydrodynamic variables; $\dot{\mathbf{q}}_{h y d}=\partial \mathbf{q}_{h y d} / \partial t ; \mathbf{q}=\left(\mathbf{q}_{h y d}, \mathbf{q}_{a c}\right)^{T}$, with $\mathbf{q}_{a c}$ being the vector of the acoustic variables (Section 3.3); and $\mathbf{F}_{a c \rightarrow h y d}=\left(0, F_{a c \rightarrow h y d}, 0,0\right)^{T}$ is the vector of forcing terms (Table 1). The hydrodynamic operator, $\mathbf{H}$, is nonlinear because of the convective derivatives and reaction term.

\subsection{Acoustic phenomena}

The acoustic variables are governed by the continuity, momentum and energy equations

$$
\begin{aligned}
& \frac{\partial \rho^{\prime \tau}}{\partial \tau}+\nabla_{\xi} \cdot\left(\langle\rho\rangle_{x} \mathbf{u}^{\prime \tau}\right)=F_{h y d \rightarrow a c_{c o n}}, \\
& \frac{\partial \mathbf{u}^{\prime \tau}}{\partial \tau}+\frac{1}{\gamma\langle\rho\rangle_{x}} \nabla_{\xi} p^{\prime \tau}=F_{h y d \rightarrow a c_{m o m}}, \\
& \frac{\partial p^{\prime \tau}}{\partial \tau}+\gamma \nabla_{\xi} \cdot \mathbf{u}^{\prime \tau}=F_{h y d \rightarrow a c_{e n}},
\end{aligned}
$$

where the spatial gradient $\nabla_{\xi}$ acts on the acoustic spatial scale, $\boldsymbol{\xi}$. This problem can be expressed as $\dot{\mathbf{q}}_{a c}-\mathbf{A q} \mathbf{q}_{a c}=\mathbf{F}(\mathbf{q})_{h y d \rightarrow a c}$, where $\mathbf{q}_{a c}=\left(\rho^{\prime \tau}, \mathbf{u}^{\prime \tau}, p^{\prime \tau}\right)^{T}$ is the vector of the acoustic variables; $\dot{\mathbf{q}}_{a c}=\partial \mathbf{q}_{a c} / \partial \tau$; and $\mathbf{F}_{h y d \rightarrow a c}=\left(F_{h y d \rightarrow a c_{c o n}}, F_{h y d \rightarrow a c_{m o m}}, F_{h y d \rightarrow a c_{e n}}\right)^{T}$ is the vector of forcing terms. The acoustic operator, $\mathbf{A}$, is linear. The nonlinearities are contained in the forcing term. State and mixture fraction equations (not shown) are required for the calculation of the heat-release terms in 2T-1S and 1T-2S (Section 3.4).

The acoustics dissipates mainly by radiation from the combustor's open boundaries and slightly in the viscous-thermal boundary layer. Nonlinear damping effects, such as vortex roll-up at sharp changes to the cross-sectional area, are not included in the current study because this study focuses on the stability of infinitesimal perturbations. In this asymptotic analysis, acoustic dissipation in the viscous-thermal boundary layer is neglected because it is of higher order. Physically, the acoustic viscous terms are negligible because (i) when the acoustic time is faster than the hydrodynamic time, 
Table 1

Terms coupling hydrodynamics to acoustics, hyd $\rightarrow a c$, and acoustics to hydrodynamics, $a c \rightarrow$ hyd. These terms depend on the multiple-scale limit: Double-time-double-space (2T-2S), double-time-single-space (2T-1S) and single-time-doublespace (1T-2S). In 2T-1S x $=\xi$, in 1T-2S $t=\tau$. The numeric subscripts of $Q_{R 0}$ and $Q_{R 1}$ refer to the orders of the heat-release asymptotic expansion.

\begin{tabular}{llll}
\hline & $2 \mathrm{~T}-2 \mathrm{~S}$ & $2 \mathrm{~T}-1 \mathrm{~S}$ & $1 \mathrm{~T}-2 \mathrm{~S}$ \\
\hline$F_{h y d \rightarrow a c_{c o n}}$ & $-\nabla_{\xi} \cdot\left(\langle\rho\rangle_{x}\left\langle\mathbf{u}^{\prime \tau}\right\rangle_{x}+\left\langle\rho^{\prime x} \mathbf{u}^{\prime x}\right\rangle_{x}\right)$ & 0 & $-\nabla_{\xi} \cdot\left(\left\langle\rho^{\prime x} \mathbf{u}^{\prime x}\right\rangle_{x}\right)$ \\
$F_{h y d \rightarrow a c_{m o m}}$ & 0 & 0 & $-1 /\langle\rho\rangle_{x} \partial / \partial t\left\langle\rho^{\prime x} \mathbf{u}^{\prime x}\right\rangle_{x}$ \\
$F_{h y d \rightarrow a c_{e n}}$ & $-\gamma\left\langle\nabla_{\xi} \cdot \mathbf{u}\right\rangle_{x}$ & $D a Q_{R 0}^{\prime \tau}$ & $D a\left\langle Q_{R 1}\right\rangle_{x}$ \\
$F_{a c \rightarrow h y d}$ & $-1 / \rho \nabla_{x} \cdot\left\langle\rho \mathbf{u}^{\prime \tau} \otimes \mathbf{u}^{\prime \tau}\right\rangle_{\tau}$ & $-1 / \rho \nabla_{x} \cdot\left\langle\rho \mathbf{u}^{\prime \tau} \otimes \mathbf{u}^{\prime \tau}\right\rangle_{\tau}$ & $-1 /(\gamma \rho) \nabla_{\xi} p^{\prime \tau}$ \\
\hline
\end{tabular}

the acoustic Reynolds number is very large and (ii) when the acoustic scale is longer than the hydrodynamics, the boundary layer scale is negligible. However, near the wall, these terms may be important and need modeling. Therefore, the viscous-thermal acoustic dissipation is modeled as a sink term in the acoustic energy equation proportional to $p^{\prime \tau}$, in a similar manner to [13]. The acoustic radiation should be modeled by impedance boundary conditions, which makes the final eigenproblem nonlinear in the eigenvalue [6]. Although the methods presented here can be applied to nonlinear eigenproblems [22], this is beyond the scope of this paper, therefore, ideal open ends are assumed, making the eigenproblem linear.

\subsection{Coupling terms}

The terms coupling the hydrodynamics to the acoustics depend on the multiple-scale limit considered (Table 1). For a more exhaustive physical understanding of these terms, Lighthill's analogies could be used, although this is not attempted here. Here, we comment on the terms that are most relevant to thermo-acoustics.

On the one hand, the hydrodynamics is an acoustic energy source through the spatially averaged dilation of the flow in the 2T-2S limit, which acts as a dipole-like source. This tends to the classic unsteady heat release in the $2 \mathrm{~T}-1 \mathrm{~S}$ and $1 \mathrm{~T}-2 \mathrm{~S}$ limits, which acts as a monopole-like source (second to last row in Table 1). Physically, the unsteady heat release only partly contributes to the acoustic energy input in the 2T-2S limit. (The unsteady heat release is calculated by using the acoustic density, temperature and mixture-fraction equations.) On the other hand, the acoustics forces the hydrodynamic momentum via the nonlinear term $-1 / \rho \nabla_{x} \cdot\left\langle\rho \mathbf{u}^{\prime \tau} \otimes \mathbf{u}^{\prime \tau}\right\rangle_{\tau}$ in the double space limits (2T-2S and 1T-2S, last row in Table 1). This term is known as the acoustic Reynolds stress [23], which, as opposed to the turbulent Reynolds stress, does not require closure because it is obtained from the acoustic solver. In the $1 \mathrm{~T}-2 \mathrm{~S}$ limit, the hydrodynamic momentum is forced through the acoustic pressure gradient that imposes a global acceleration.
In compact form, the coupled thermo-acoustic problem (Fig. 1), governed by (6)-(12), reads

$\dot{\mathbf{q}}-\mathbf{T}(\mathbf{q})=\mathbf{F}$,

where $\mathbf{T}(\mathbf{q})$ is the nonlinear thermo-acoustic operator, and $\mathbf{F}=\left(\mathbf{F}_{a c \rightarrow h y d}, \mathbf{F}_{h y d \rightarrow a c}\right)^{T}$.

\section{Linearization, stability and intrinsic sensitivity}

In linearization, we typically assume that the amplitudes of the hydrodynamic variables are $\sim$ $O(1)$ and those of the acoustic variables are $\sim O(\varepsilon)$. (This perturbation parameter $\varepsilon$ has nothing to do with the multiple-scale perturbation parameter $\epsilon$.) Physically, we regard the acoustics as a perturbation field on top of the hydrodynamic flow. However, we show that different multiple-scale limits cause different linear behaviors. The hydrodynamic variables are split as $\mathbf{q}_{h v d}=\overline{\mathbf{q}}_{h v d}+\varepsilon \mathbf{q}_{h v d, 1}$, where $\overline{\mathbf{q}}_{h v d}$ $\sim O(1)$ is the steady mean flow calculated from numerical simulations or experiments and $\varepsilon \mathbf{q}_{h y d, 1}$ is the low-frequency large-scale organized motion. Therefore, the perturbation hydrodynamic vector, $\mathbf{q}_{h y d, 1}$, and acoustic vector, $\mathbf{q}_{a c}$, are of the same or$\operatorname{der} \varepsilon$ but act at different scales. When linearized, the thermo-acoustic problem can be expressed in compact form as $\dot{\mathbf{q}}=\mathbf{J q}$, where

$$
\mathbf{J}=\left[\begin{array}{cc}
\left(\frac{\delta \mathbf{H}}{\delta \mathbf{q}_{h y d}}+\frac{\delta \mathbf{F}_{a c \rightarrow h y d}}{\delta \mathbf{q}_{h y d}}\right) & \frac{\delta \mathbf{F}_{a c \rightarrow h y d}}{\delta \mathbf{q}_{a c}} \\
& \left(\mathbf{A} \mathbf{F}_{h y d \rightarrow a c}\right. \\
\delta \mathbf{q}_{h y d} & \left(\mathbf{A}+\frac{\delta \mathbf{F}_{h y d \rightarrow a c}}{\delta \mathbf{q}_{a c}}\right)
\end{array}\right] .
$$

The Jacobian operator is the functional derivative of the thermo-acoustic operator, $\mathbf{J}=\delta \mathbf{T} / \delta \mathbf{q}$, and is evaluated at the base flow $\overline{\mathbf{q}}_{h y d}$. When we linearize the double-time limits, $2 \mathrm{~T}-2 \mathrm{~S}$ and $2 \mathrm{~T}-1 \mathrm{~S}$, the acoustic Reynolds stress, which is the term coupling the acoustics to the hydrodynamics, vanishes. The linear dynamics is only one-way coupled because the coupling term $\delta \mathbf{F}_{a c \rightarrow h y d}=O\left(\varepsilon^{2}\right)$ is negligible in (14). This is because, when there are two time scales (the acoustic time being faster than the convective time), the acoustics are driven by the hydrodynamics but do not affect it. Physically, this is because the influence of the acoustics averages to zero over the long time scale of the hydrodynamics. This is equivalent to one of the mechanisms described in [1] in which, if one considers 
perturbations convecting at uniform speed along a long flame such that there are many oscillations along the flame, most of the heat release perturbations cancel out, causing the flame to behave as a low pass filter. From this two-scale argument, a thermo-acoustic oscillation is more likely to exist when the time scales are the same. In a classic picture of a thermo-acoustic instability, the two time scales are indeed the same [12]. In fact, when only one time scale is modeled, as in 1T-2S, the thermoacoustic system is two-way linearly coupled because $\delta \mathbf{F}_{a c \rightarrow h y d}=O(\varepsilon)$, i.e., there is a non-trivial interaction between hydrodynamic and thermo-acoustic stability, as shown in Section 5. Therefore, we use the 1T-2S limit from now on. By using modal transformations, $\mathbf{q}_{1}=\hat{\mathbf{q}} \exp (\sigma t)$, the resulting direct thermo-acoustic eigenproblem becomes $\sigma \hat{\mathbf{q}}=$ $\mathbf{J} \hat{\mathbf{q}}$, where $\sigma$ is the complex eigenvalue. The adjoint modal transformation, $\mathbf{q}_{1}^{+}=\hat{\mathbf{q}}^{+} \exp \left(-\sigma^{*} t\right)$, yields the adjoint eigenproblem $\sigma^{*} \hat{\mathbf{q}}^{+}=\mathbf{J}^{H} \hat{\mathbf{q}}^{+}$, where ${ }^{*}$ is the complex conjugate and ${ }^{H}$ denotes the complex transpose. The adjoint eigenfunctions provide the receptivity to open-loop forcing. For example, the most receptive zone due to flow velocity disturbances is the shear-layer where the two streams mix [24].

The eigenfunctions and their adjoints can be combined to predict the first-order eigenvalue-drift caused by a spatially localized perturbation to the system, $\delta \mathbf{J}$, as

$\delta \sigma=\hat{\mathrm{q}}_{i}^{+H} \delta \mathbf{J}_{i j} \hat{\mathrm{q}}_{j}$,

where the indices denote the components of the matrix/vector arrangement of the continuous operators, i.e. $i, j=\rho, \mathbf{u}, T, Z, \rho^{\prime \tau}, \mathbf{u}^{\prime \tau}, p^{\prime \tau}$. The eigenfunctions are normalized such that $\hat{\mathbf{q}}^{+H} \hat{\mathbf{q}}=1$. Eq. (15) was introduced in thermo-acoustics to calculate the effect of external feedback mechanisms, such as a hot wire, and design-parameter changes, such as the flame inlet geometric ratio, in the stability [7-9] via appropriate models for the thermo-acoustic perturbation $\delta \mathbf{J}$. We introduce the concept of intrinsic sensitivity, extending the structural sensitivity used in $[7,18]$, defined as the eigenvalue's sensitivity to intrinsic physical mechanisms. Here, the perturbation operator of the eigenvalue's sensitivity formula (15) is the Jacobian itself, i.e., $\delta \mathbf{J}=\varepsilon \mathbf{J}$. This enables us to identify the regions of the flow where the hydrodynamic and acoustic subsystems are active and quantify how they affect the overall thermo-acoustic stability. When the flow is laminar, the real part of (15) provides a map showing the regions to which the thermo-acoustic stability is most sensitive to the $j$ th variable through the $i$ th equation. Likewise, the imaginary part of (15) shows the regions to which the thermo-acoustic angular frequency is most sensitive.

\section{Application to a coaxial jet combustor}

We use the 1T-2S limit because it enables the two-way linear coupling between the hydrodynamic and acoustic subsystems. The problem is cast in weak form in cylindrical coordinates and Fouriertransformed in the azimuthal direction exploiting the axisymmetry. We discretize the equations with a finite element method (http://www.freefem.org/ff + $+/$ ) and solve the eigenproblem and its adjoints with a shift-invert method with ARPACK (http://www. caam.rice.edu/software/ARPACK/). We use a Discrete Adjoint approach $[7,25]$ to ensure that the adjoint spectra match the complex conjugate of the direct spectra to machine precision. The code was validated against the solutions of [18] and the spectra matched with an error smaller than $0.5 \%$. To test our method, we use the coaxial jet combustor of $[14,15]$, which is schematically shown in Fig. 1. The mean flow has no swirl and we investigate the azimuthally symmetric (non-spiralling) Fourier component, whose hydrodynamic mode can couple with longitudinal acoustic oscillations creating a thermo-acoustic instability. The mean-flow boundary conditions are plug-flow at the inlet, no-slip flow at the walls and convective outflow at the outlet. As for the stability equations, homogeneous Dirichlet boundary conditions are prescribed on the low-Mach number equations everywhere except for the outlet, where homogeneous Neumann conditions are imposed. The acoustic pressure is zero at the ends (open-ended combustor) and the acoustic velocity is zero at the rigid walls. After performing convergence tests, we choose a discretization with 90,429 triangles and 45,792 vertices for both the hydrodynamics and acoustics. The case presented has the following parameters: $R e=100$ (laminar flow and steady solution); $P r=S c=0.7 ; \kappa=0.01$; $s=2 ; \beta=3 ; S_{1}=7 ; S_{2}=6 ; D a=10^{6}$. These conditions correspond to a methane/air flame. A similar flame was studied by [17] in an unconfined domain without inlets. In this laminar case, the mean flow (Fig. 2) is a steady solution of the governing Eqs. (6)-(9) and it is labelled the base flow. The base flow is obtained by time-marching a structured Large-Eddy Simulation low-Mach number code [15] until the steady solution is obtained. The chemistry is solved with a Flamelet Progress Variable Method [15].

On the one hand, the combusting hydrodynamic flow, when the coupling forcing terms are set to zero, is globally stable. The dominant eigenvalue is $\sigma=-0.100+\mathrm{i} 1.041$. The radial velocity and temperature eigenfunctions are depicted in Fig. 3a and b, respectively. On the other hand, the acoustics, when the linearized low-Mach number flow is not coupled through forcing terms, are stable because of the acoustic damping, although close to neutral stability. The eigenvalue is $\sigma=-0.00031+\mathrm{i} 0.630$. As a check, the nondimensional acoustic angular frequency $\sigma_{i}=0.630$ 
(a) $\bar{u}_{x}$

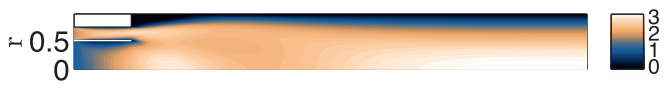

(b) $\bar{u}_{r}$

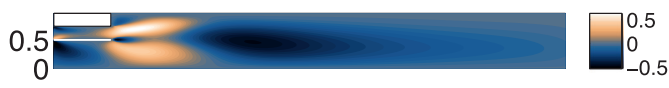

(c) $\bar{T}$

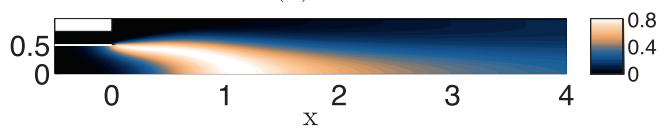

Fig. 2. Base-flow (a) axial velocity, (b) radial velocity and (c) temperature.

is close to the non-dimensionalized first duct resonance, $\left(2 \pi \tilde{c}_{0} / 2 L\right) /\left(\tilde{c}_{0} / 2 R_{1}\right)=0.698$, where $L=9 R_{1}$ is the combustor's length (Fig. 1). The acoustic mode is longitudinal (Fig. 3c), although the effect of the radial acoustic motion is not negligible at the inlet (Fig. 3d).

When two-way coupled, the low-Mach number flow and acoustics become unstable, with dominant eigenvalue $\sigma=0.102+\mathrm{i} 0.731$. The angular frequency is close to the acoustic frequency; the instability is driven by acoustic effects because the unstable mode is primarily acoustic. In this stability framework, the hydrodynamics is simulated and interacts with the acoustics. This overcomes the limitations of simple thermo-acoustic models, in which all the information from the hydrodynamics is typically encapsulated in response functions [4,5], $n-\tau$ models and other models for the flame convective speed [1].

We can study the effect that the acoustics has on the hydrodynamic stability, which is exactly given by the intrinsic sensitivity map $\delta \sigma / \varepsilon=\hat{\mathbf{q}}_{h y d}^{+H} \mathbf{J}_{12} \hat{\mathbf{q}}_{a c}$. This is a spatial function and $\mathbf{J}_{12}$ is the coupling operator from the acoustics to the hydrodynamics (top-right component of the matrix (14)) through the acoustic pressure gradient (bottom-right term in Table 1). This formula quantifies how much a small perturbation to the acoustic field $\sim \varepsilon \mathbf{J}_{12} \hat{\mathbf{q}}_{a c}$ changes the thermo-acoustic eigenvalue of the coupled system through hydrodynamic physical process $\left(\hat{\mathbf{q}}_{h y d}^{+H}\right)$. The real part of this quantity is shown in Fig. 4a. We note that the highest sensitivity straddles the recirculation region at the top left corner. The acoustics is acting as an extra feedback momentum source, enhancing the hydrodynamic sensitivity, which is, indeed, often close to the recirculation boundary [25]. (From a hydrodynamic standpoint, in the $1 \mathrm{~T}-2 \mathrm{~S}$ linear limit, the acoustics cannot be viewed as an external forcing.) Changes in the strength of the coupling from the acoustics to the hydrodynamics here will have the most influence on this mode, i.e., the mode is very sensitive to the coupling in this region. However, this is only one component of the intrinsic sensitivity and the coupling from acoustics to hydrodynamics is not the dominant mechanism. The maximum growth rate drift is $\delta \sigma_{r} \sim O\left(10^{-4}\right)$.

Following the same line of reasoning, we study the effect that the hydrodynamics has on the stability through acoustic mechanisms, whose intrinsic sensitivity is $\delta \sigma / \varepsilon=\hat{\mathbf{q}}_{a c}^{+H} \mathbf{J}_{21} \hat{\mathbf{q}}_{\text {hyd }}$, where $\mathbf{J}_{21}$ is the coupling term from the hydrodynamics to the acoustics (bottom-left component of the matrix (14)). This formula quantifies how much a small perturbation to the hydrodynamic field $\sim \varepsilon \mathbf{J}_{21} \hat{\mathbf{q}}_{\text {hyd }}$ changes the thermo-acoustic eigenvalue of the coupled system through acoustic physical process $\left(\hat{\mathbf{q}}_{a c}^{+H}\right)$. This map is depicted in Fig. 4b. The maximum value is $\delta \sigma_{r} \sim O\left(10^{-2}\right)$. The region of high sensitivity straddles the stoichiometric line, where

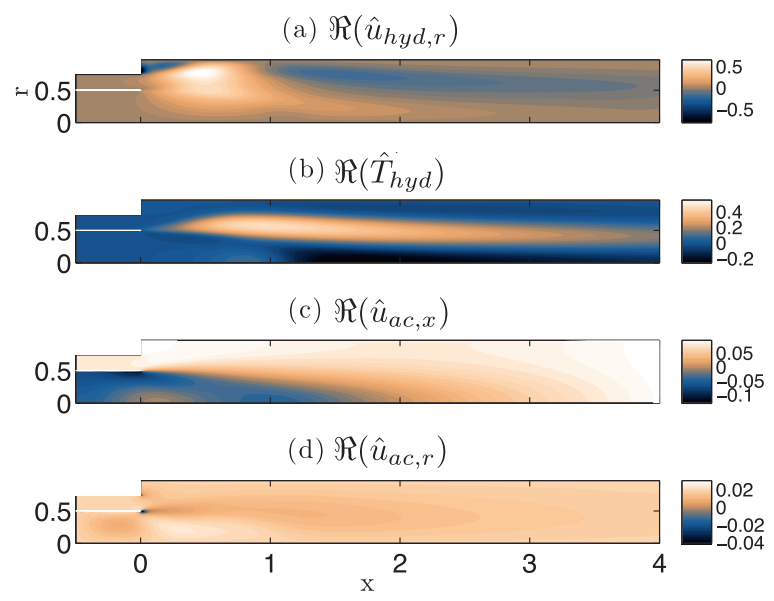

Fig. 3. Real part of (a,b) hydrodynamic and (c,d) acoustic eigenfunctions. 

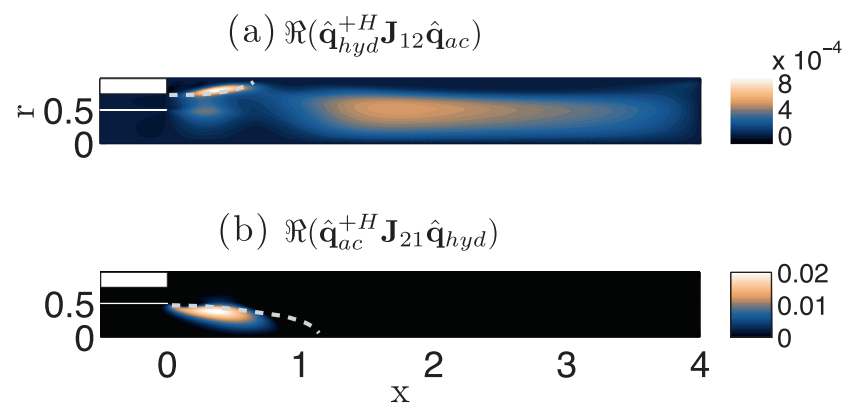

Fig. 4. Growth-rate sensitivity (a) to intrinsic hydrodynamic feedback feeding the momentum equation, the maximum straddles the recirculation-boundary (upper dashed line) and (b) sensitivity to the hydrodynamics through acoustic mechanisms, the maximum straddles the stoichiometric line (lower dashed line).

most of the heat is released by the flame. This shows that a small change in the coupling from hydrodynamics to acoustics causes a larger stability drift, i.e. $\delta \sigma_{r} \sim O\left(10^{-2}\right)$, than a small change in the coupling from the acoustics to the hydrodynamics, i.e. $\delta \sigma_{r} \sim O\left(10^{-4}\right)$. In other words, small changes of the strength of the hydrodynamic feedback (i.e., coupling) greatly change the flame response to acoustic perturbations which, in turn, have significant influence on the thermo-acoustic stability. In the limit of classic one-way coupled thermo-acoustic models, this result is consistent with the diffusion-flame structural sensitivity and Rayleigh Index analysis of [8]. In this paper, we have focused on the terms coupling the hydrodynamics to the acoustic energy equation because they are directly connected to the unsteady heat release. The investigation of the remaining coupling terms in the acoustic continuity and momentum equations (Table 1) is left for future studies. This can be directly tackled with the intrinsic structural sensitivity concept introduced in Eq. (15).

\subsection{Effect of the global acceleration term}

In the Rijke-tube configuration of [12], the coupling term $F_{a c \rightarrow h y d}$, also known as the global acceleration term $[11,12]$, was shown to have a noticeable destabilizing effect on the thermo-acoustic stability. In our configuration, as the coupling strength from the acoustics to the hydrodynamics increases, the growth rate decreases non-monotonically (Fig. 5a). This is physically explained via the Rayleigh Criterion [2]. By substituting the acoustic eigenfunctions into the definition of acoustic energy, $E_{a c}$, and neglecting damping to a first approximation [8], the acoustic-energy rate of change is given by the integral of the Rayleigh Index over the acoustic domain, $\sigma_{r} E_{a c}=1 / 4 \Re\left(\int_{V} \hat{p}^{\prime \tau *} \hat{\mathcal{Q}} \mathrm{d} \xi\right)$; where $\hat{\mathcal{Q}}$ is the acoustic heat release given by $F_{h y d \rightarrow a c_{e n}}$ in Table 1 . Both $\hat{p}^{\prime \tau}$ and $\hat{\mathcal{Q}}$ are implicit function of the global acceleration term $F_{a c \rightarrow h y d}$, and so is $\sigma_{r} E_{a c}$. By splitting the contribution as $\sigma_{r} E_{a c}=\left(\sigma_{r} E_{a c}\right)_{F=0}+$ $\delta\left(\sigma_{r} E_{a c}\right)$, where the subscript $F=0$ is the contribution when $\left\|F_{a c \rightarrow h y d}\right\|=0$ and $\delta(\cdot)$ is the contribution when $\| F_{a c} \rightarrow$ hyd $\|>0$, we see that the effect that the global acceleration term has on the thermo-acoustic stability depends on the phase of $\delta\left(\hat{p}^{\prime *} \hat{\mathcal{Q}}\right)$. In our configuration this is negative (Fig. 5b), i.e., the acoustic-energy rate of change decreases, whereas we expect that in [12] this energy excess was positive. We infer that, in general, the global acceleration term can have either a stabilizing or destabilizing effect because the feedback can be positive or negative depending on the phase (the eigenmodes are complex), which, in turn, depends on the configuration.

\section{Conclusions}

In the first part of this paper, we propose a methodology to calculate thermo-acoustic stability and sensitivity. By using different time and spatial scales for hydrodynamic and acoustic phenomena, we formulate a three-dimensional thermo-acoustic stability model in which the hydrodynamic field two-way interacts with the acoustics. Only a lowMach number solver and an acoustic solver are needed. Three multiple scale limits are revisited and the two-way coupled system is linearized for stability analysis. It is shown that only when the acoustics evolve at larger spatial scales than the hydrodynamics but at the same time scale (1T-2S limit), the linearized mutual interaction is two-way coupled. Using this limit, we define an eigenproblem and show how to study the sensitivity of the stability with an adjoint method. The intrinsic sensitivity identifies the regions and quantifies the effect that different subsystems have on the overall stability, which is suitable for multi-physical problems such as thermo-acoustics. In the second part, we apply this methodology to a coaxial jet combustor. We show that, although the hydrodynamic and acoustic subsystems are stable if considered separately, 
(a) $\sigma_{r}$
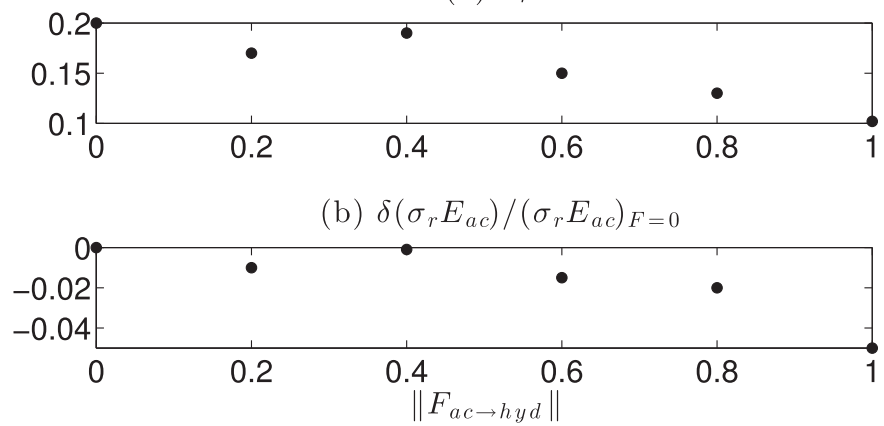

Fig. 5. Effect of the coupling term $F_{a c \rightarrow h y d}$ on (a) the thermo-acoustic growth rate and (b) Rayleigh Criterion as a function of its normalized strength.

they become unstable when coupled together. The exact effect that the acoustics has on the hydrodynamic stability is quantified and physically interpreted as an extra momentum source enhancing the region of high hydrodynamic sensitivity. The hydrodynamics greatly influences the overall thermoacoustic stability in regions of large heat release. The term coupling the acoustics to the hydrodynamics, known as the global acceleration term, can have either a stabilizing effect, as shown here, or a destabilizing effect, as shown in [12], on the thermoacoustic stability. It was shown that this behavior is due to its contribution to the Rayleigh Criterion, which is not a monotonic function of the globalacceleration strength.

This study shows that the relatively cheap combination of a low-Mach number solver, an acoustics solver, and adjoint sensitivity analysis can (i) avoid fully compressible calculations and (ii) reveal the most influential intrinsic mechanisms in thermo-acoustic instability. This technique can readily be extended to more complex geometries and passive control.

\section{Acknowledgments}

The following sources of funding are gratefully acknowledged: the 2014 CTR Summer Program, ERC Project ALORS 2590620 and NASA grant \#NNX10CF79P. L.M. also acknowledges the Royal Academy of Engineering Research Fellowships scheme. L.M. is grateful to Dr. C. Balaji and Prof. S.R. Chakravarthy for helpful discussions.

\section{References}

[1] T. Lieuwen, Unsteady Combustor Physics, Cambridge University Press, 2012.

[2] L. Rayleigh, Nature 18 (1878) 319-321.

[3] S.R. Chakravarthy, O.J. Shreenivasan, B. Boehm, A. Dreizler, J. Janicka, J. Acoust. Soc. Am. 122 (1) (2007) 120-127.
[4] W. Polifke, A. Poncet, C.O. Paschereit, K. Döbbeling, J. Sound Vib. 245 (3) (2001) 483-510.

[5] N. Noiray, D. Durox, T. Schuller, S. Candel, J. Fluid Mech. 615 (2008) 139-167.

[6] F. Nicoud, L. Benoit, C. Sensiau, T. Poinsot, AIAA J. 45 (2) (2007) 426-441.

[7] L. Magri, M.P. Juniper, J. Fluid Mech. 719 (2013) 183-202.

[8] L. Magri, M.P. Juniper, J. Fluid Mech. 752 (2014) 237-265.

[9] L. Magri, M.P. Juniper, Int. J. Spray Combust. Dyn. 6 (3) (2014) 225-246.

[10] G. Rigas, N.P. Jamieson, L.K.B. Li, M.P. Juniper, J. Fluid Mech. 787 (2016) R1.

[11] J.P. Moeck, M. Oevermann, R. Klein, C.O. Paschereit, H. Schmidt, Proc. Combust. Inst. 32 (1) (2009) 1199-1207.

[12] S. Mariappan, R.I. Sujith, J.Fluid Mech. 680 (2011) 511-533.

[13] C. Balaji, S.R. Chakravarthy, in: 18th International Congress on Sound and Vibration 2011 (ICSV 18), Rio de Janeiro, Brazil, 2011. July

[14] F.K. Owen, L.J. Spadaccini, C.T. Bowman, Proc. Combust. Inst. 16 (1) (1977) 105-117.

[15] C.D. Pierce, P. Moin, J. Fluid Mech. 504 (2004) 73-97.

[16] L. Magri, Y.C. See, M. Ihme, M.P. Juniper, Center for Turbulence Research Summer Program, Center for Turbulence Research, Stanford, USA, 2014, pp. 199-208.

[17] J.W. Nichols, P.J. Schmid, J. Fluid Mech. 609 (2008) 275-284.

[18] U.A. Qadri, G.J. Chandler, M.P. Juniper, J. Fluid Mech. 775 (2015) 201-222.

[19] B. Müller, J. Eng. Math. 34 (1998) 97-109.

[20] R. Klein, ESAIM Math. Model. Numer. Anal. 39 (3) (2005) 537-559.

[21] T. Poinsot, D. Veynante, Theoretical and numerical combustion, 2nd, R. T. Edwards, 2005.

[22] M.P. Juniper, L. Magri, M. Bauerheim, F. Nicoud, Center for Turbulence Research Summer Program, Center for Turbulence Research, Stanford, USA, 2014, pp. 189-198.

[23] J. Lighthill, J. Sound Vib. 61 (3) (1978) 391-418.

[24] B. Emerson, T. Lieuwen, M.P. Juniper, J. Fluid Mech. 788 (2016) 549-575.

[25] P. Luchini, A. Bottaro, Annu. Rev. Fluid Mech. 46 (2014) 1-30. 